\title{
Ecological Site Development: Accelerating the Effort
}

\author{
By Jamin Johanson and Joel Brown
}

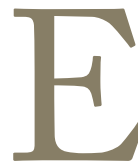
cological site descriptions (ESDs) have unmatched potential as rangeland classification tools with practical management applications. Yet despite their great potential, the majority of ESDs currently lack sufficient detail to satisfy the needs of land managers, researchers, and other ESD users. Fortunately, however, a notable increase in the tools, resources, and support needed for ESD development promises to improve the concepts, delivery, and application of this important management resource. ${ }^{1}$

An ecological site is a distinctive type of land that differs from other land in 1) its ability to produce distinctive kinds and amounts of vegetation and 2) its response to management actions and natural disturbance. Simply put, ecological sites provide a meaningful way to interpret the landscape. The ESD is a document that describes the soil, climate, physiography, and plant community dynamics that characterize an ecological site. The Natural Resources Conservation Service (NRCS) adopted ecological sites to replace the more livestock-oriented range sites in $1997 .{ }^{2}$ Unlike the old range site descriptions, ESDs contain information about the plant community dynamics as described through state-and-transition models (STMs), wildlife habitat, hydrology, reference conditions for indicators of rangeland health, and other relevant information when available.

Ecological sites were first recognized by the Bureau of Land Management and the Forest Service in 2005. In the short time since, ESDs have gained acceptance as an ideal framework for many range management applications, including but not limited to the following: evaluating rangeland health, establishing monitoring sites, facilitating communication within and among diverse groups, understanding plant community dynamics through utilization of STMs, and planning and evaluating range improvement projects. As the list of ESD uses and users continues to grow, the production of reliable ESDs will require more substantial inputs and earnest collaboration from range management professionals devoted to developing this important resource.

At the SRM annual meeting in Denver, Colorado, in 2010, NRCS leadership announced an effort to "accelerate" the ESD development process using a streamlined workflow that employs quality control, quality assurance, and increased staffing measures. The term "acceleration" implies an increase in activity, speed, and progress. Acceleration of ESD development requires every individual part to function efficiently and synchronously with the other system components to generate maximum utility.

\section{ESD Development Workshop}

In an effort to streamline some of the common day-to-day tasks associated with ESD development, several regional, national, and field-level ESD specialists organized and contributed their ideas for an ESD development workshop at the 2011 SRM annual meeting in Billings, Montana. The workshop provided an opportunity for ESD developers to teach one another about many of the innovative ESD development tools being employed in different parts of the United States. The purposes of the workshop were to 1) provide an overview of the ESD development effort on a national and state level, 2) provide training and present tools that improve the accuracy and efficiency of ESD development, and 3) facilitate communication and establish professional networks among ESD developers.

The workshop consisted of three parts: an overview of ESD efforts, ESD development tools, and advanced ESD development methods.' It was moderated by Joel Brown, NRCS range ecologist from Las Cruces, New Mexico, who introduced the session by defining ESDs and their importance. $\mathrm{He}$ summarized the current state of the ESD acceleration effort: 1) ESD development requires strong commitment from government agencies, 2) technical field staff perceive ESDs as relevant management tools, 3) interagency coordination is not yet functional and will require substantial advancements in the ESD development process, and 4) progress will occur when inclusive training and participation opportunities are made available to all ESD developers and users. These points were established by ESD leaders and developers nationwide at an ESD development pilot

'The presentations from all three parts of the workshop can be found online at http://jornada.nmsu.edu/esd/workshops. 
workshop held just 3 months prior in Las Cruces, New Mexico.

\section{Part I: Overview of ESD Efforts}

The first part of the workshop provided an overview of the ESD development effort at the state and national levels. Speakers were selected from the NRCS national and regional leadership, and a succession of themes emerged regarding the current condition of the overall ESD development effort. First, the development of an estimated 18,000 ESDs covering the entire United States is an enormous task. Accelerated ESD development will require not only additional resources, but an entirely new approach to doing business. Even so, there is leadership support for ESD acceleration. Terrell Erickson, director of the NRCS Ecological Sciences Division, announced that the draft Interagency Rangeland Ecological Site Manual is available for public comment, and a national interdisciplinary team has been established to guide the ESD development effort. In addition, regional and field office staff positions have been created to increase the rate of production.

Homer Sanchez, NRCS South Technical Center rangeland specialist, presented a proposed ESD development workflow plan that promotes high standards through quality control and quality assurance. However, despite these organizational improvements within the NRCS, the technical support for accelerated ESD development is currently in the beginning stages. It was noted that the new ESD development system will likely mirror the current NRCS soil survey in terms of its capabilities and continuity.

Pat Shaver, NRCS West Technical Center rangeland specialist, introduced the need for communication and collaboration in order to correlate ecological sites across political boundaries (i.e., states and soil survey areas). Which leads to the final and overarching theme from the first part of this workshop: there is a need for improved communication and collaboration among agencies, states, regional offices, and all other partners to clarify standards, resources, responsibilities, limitations, and other details pertaining to the technical components of day-to-day ESD development. The questionand-answer session concluding Part I was also dominated by the topic of communication and collaboration among ESD developers to optimize the quality of ESD products.

\section{Part II: ESD Development Tools}

Parts II and III of this workshop focused on specific ESD development tools that improve the establishment and refinement of ecological site concepts. Speakers in Part II presented data extraction tools to help characterize the soils, physiography, climate, and spatial extent of an ecological site using broadly accessible datasets. These speakers were NRCS rangeland specialists that use these tools in their daily ESD development responsibilities. Justin Clary from Fort Worth, Texas, showed three ways to develop the soil and physiography sections of ESDs using existing soil survey data. He provided step-by-step instructions to obtain ecological site-specific soil survey data from the National Soils Information System, Soil Data Mart," and Soil Data Viewer.iii $\mathrm{He}$ also provided a comparison of the strengths and limitations of each method. Jacob Owens from Richfield, Utah, followed with a presentation on creating ecological site maps and using shapefiles to extract spatial data. The map creation process has dozens of tedious steps, so an alternative automated process was also presented. Though much easier to use and significantly less time-consuming, the automated process is copyrighted and must be purchased if it is to be widely distributed for ESD development. Sarah Quistberg and Jamin Johanson from Ogden and Richfield, Utah, concluded the session with different methods of obtaining site-specific climate data. Both methods depend on accurate ecological site maps to extract data from existing datasets. Sarah presented a tool developed by Michael Carpinelli, former rangeland specialist in New Mexico, which summarizes climate station data from the Western Regional Climate Center. ${ }^{\text {iv }}$ Jamin shared a tool that extracts and summarizes modeled climate data (Parameter-Elevation Regressions on Independent Slopes Model [PRISM] $]^{v}$ ) for sites that lack climate station data.

It became evident from these presentations that sufficient information is available to refine or develop initial soil- and climate-based site concepts for most existing ESDs; however, the complex ecological knowledge needed to develop quality STMs is not readily available in any particular database. Additionally, it was apparent that the establishment of data standards would be more appropriate than specific protocols at the present time, since different methods can be used to obtain a high standard of relevant information and protocols may stifle further innovation. However, if ESD acceleration is to be most efficient, it is recommended that some of these data extraction tools be automated and distributed for use throughout the country. In particular, there is a glaring need to automate ecological site mapping, since many of the tools presented in Parts II and III require accurate spatial data to extract important site-specific information. This reflects a more general need for ecological site maps that supplement on-the-ground ecological site identification and enable ESD developers, researchers, and partners to plan site-specific projects.

\section{Part III: Advanced ESD Development Methods}

The methods presented in Part III differed from those in Part II in their degree of complexity. Jamin Johanson presented a computer model developed by Gale Dunn, soil

\footnotetext{
iSoil Data Mart can be accessed at http://soildatamart.nrcs.usda.gov/. iii Soil Data Viewer can be accessed at http://soils.usda.gov/sdv/.

iv Climate data from the Western Regional Climate Center can be accessed at http://www.wrcc.dri.edu/.

vPRISM data can be accessed at http://www.prism.oregonstate.edu/.
} 
scientist for the Agricultural Research Service in Fort Collins, Colorado, that generates plant community production curves based on soil, climate, and plant physiological attributes. Ken Spaeth, NRCS South Technical Center rangeland specialist, demonstrated one way to describe the hydrology of an ecological site using the Range Hydrology and Erosion Model (RHEM tool). ${ }^{\text {vi }}$ The RHEM tool estimates runoff, infiltration, and erosion based on ecological site parameters such as soil texture, slope, precipitation, and kind and amount of vegetation cover. Kendra Moseley, NRCS West Technical Center rangeland specialist, provided tips for organizing and interpreting complex plant community datasets. She emphasized the importance of data entry into a database with querying capabilities. As an example of successful interdisciplinary collaboration, Kirt Walstad, NRCS rangeland specialist from Bozeman, Montana, showed one way to develop dichotomous ecological site keys. Kirt explained the rigorous process of communicating and collaborating to define the soil-geomorphic and climatic properties of all ecological sites in order to develop a regional ecological site key. The workshop concluded with two examples of plant community mapping using remote sensing. Doug Ramsey, Utah State University professor, showed the utility of mapping common plant species with remote sensing to delineate ecological sites at a finer scale than that of higher order soil surveys. Alex Hernandez, PhD candidate from Utah State University, showed how remote sensing can be used for STM development by tracking changes in plant community composition over a 30-year period.

The complexity of the methods presented in Part III and the ecological knowledge required for STM development reveals the critical need for well-trained range professionals, capable of integrating information from various sources, to continue to develop useful tools and accelerate ESD development. It also highlights the importance of collaborating across disciplines to incorporate technology and expertise into the ESD development process. When equipped with the best tools and resources available, such as those presented at this workshop, well-trained range

viRHEM tool can be accessed at http://apps.tucson.ars.ag.gov/rhem/. professionals will become the engine of the ESD acceleration effort.

\section{Sustained Acceleration Efforts}

This ESD development technical workshop helped boost the ESD acceleration effort by providing some initial training of new technologies, as well as by establishing new communication networks for ESD developers. It also clarified the strengths and weaknesses of the ESD acceleration effort in its current state. Within the framework established by strong national and regional leadership, future technical workshops are critical to provide examples of accelerated ESD development, share tools, explore collaborative opportunities, improve communication, and clarify ESD development standards. To address some of these critical needs, a follow-up ESD development technical workshop was held on 28 January at the 2012 international SRM meeting in Spokane, Washington. The purposes of the workshop were to 1) provide an overview of the history, concepts, and applications of ESDs and STMs; 2) provide examples of successful STM development using a variety of data sources; 3) provide training and access to tools available for accelerated ESD development; 4) provide examples of successful management applications of ESD and STM concepts; and 5) create networking opportunities and generate discussion among ESD specialists.

These and other ESD development workshops provide momentum for the ESD acceleration effort, which, when realized, will be a great benefit to the ever-advancing range profession.

\section{References}

1. Bestelmeyer, B. T., And J. R. Brown. 2010. An introduction to the special issue on ecological sites. Rangelands 32:3-4.

2. US Department of Agriculture Natural Resources Conservation Service. 1997. National range and pasture handbook. Washington, DC, USA: US Department of Agriculture. $472 \mathrm{p}$.

Authors are Range Specialist, USDA-NRCS, Richfield, UT 84701, USA, jamin.johanson@ut.usda.gov (Johanson); and Rangeland Ecologist, USDA-NRCS Jornada Experimental Range, Las Cruces, NM 88003, USA (Brown). 